\section{Eye movements and their functions in everyday tasks}

Department of Psychology, University of Essex,

Colchester, UK

Correspondence:

T Foulsham, Department of Psychology, University of Essex, Wivenhoe Park, Colchester CO4 3SQ, UK Tel. +44 (0)1206 874159; Fax: +44 (0)1206 873801 E-mail: foulsham@ essex.ac.uk

Received: 7 October 2014 Accepted in revised form: 8 October 2014 Published online: 14 November 2014

Paper presented at the Cambridge Ophthalmological Symposium 2014.

\begin{abstract}
Human saccades and fixations have numerous functions in complex everyday tasks, which have sometimes been neglected in simple experimental situations. In this review I describe some of the characteristics of eye movement behaviour during realworld interactions with objects, while walking in natural environments and while holding a conversation. When performing real-world actions and walking around the world, we fixate relevant features at critical time points during the task. The eye movements between these fixations are planned and coordinated alongside head and body movements, often occurring a short time before the corresponding action. In social interactions, eye movements are both a mechanism for taking in information (for example, when looking at someone's face or following their gaze) and for signalling one's attention to another person. Thus eye movements are specific to a particular task context and subject to high-level planning and control during everyday actions.

Eye (2015) 29, 196-199; doi:10.1038/eye.2014.275; published online 14 November 2014
\end{abstract}

\section{Introduction}

From the point of view of the retina, the function of saccadic eye movements in humans is clear. By frequently orienting our eyes to different points in the visual field, we ensure that the high acuity of central vision is available wherever we need it. This review focuses on saccades and fixations, which can be made voluntarily, and which are an essential part of vision in most animals. ${ }^{1}$ Indeed, evidence from a patient with ophthalmoplegia suggests that even when eye movements are not possible, saccade-like head movements are made in order
T Foulsham

to sample the environment in a similar way. ${ }^{2}$ Saccadic eye movements reflect the moment-tomoment positioning of the fovea, and hence the current input to the visual system. As a result, the location and duration of fixations have become important measures of visual attention in experimental psychology and cognitive neuroscience. $^{3}$

Although much is now known about the way that humans move their eyes in controlled experiments, it is more of a challenge to determine the characteristics and function of eye movements in natural tasks. This challenge is partly technological, and it is only in the last two or three decades that mobile recording systems have enabled eye tracking in participants who are free to move around. 4,5 When considering studies of eye movements in everyday actions, it becomes clear that we move our eyes in many different ways, and that the function of these movements can only be understood in the context of a particular task. Yes, we fixate to retrieve information from a particular location. However, the control and timing of saccades and associated head and body movements vary widely with the task. I will give some examples of the tasks in which eye movements have been described, before drawing some conclusions on their functions in each context.

\section{Acting on objects}

In several pioneering papers, Land, Hayhoe and colleagues have reported detailed recordings of gaze behaviour during complex everyday tasks. ${ }^{6-8}$ These tasks (making tea and sandwiches) can be broken down into a stereotyped sequence of actions, often involving the manipulation of one or more objects to achieve a goal. For example, when making tea one must fill the kettle, reach for the milk and so on. Different people were rather consistent in the way that they moved their eyes at each 
stage. During sandwich-making, for example, they might look at a jar of peanut butter, reach for it and open the jar, simultaneously looking back to the slice of bread on the plate. Detailed inspection of these recordings showed that the relative timing of body, hand and eye is systematic, and that fixations on irrelevant objects were very rare.

Several general principles have emerged from this research. First, Hayhoe and colleagues noted that the majority of fixations were directed at the information necessary for the current action (so called 'just-in-time' selection). Thus fixations pick up information as and when it is needed, rather than people representing this information in memory. Second, gaze tends to shift to an object between 0.5 and $1 \mathrm{~s}$ before an action (such as reaching with the hand) begins. Moreover, the eyes normally move on to the next required object before that action is completed. The fixation, therefore, must provide information about the target for the reach, such as the object's location and the grip needed, but it is normally not necessary to provide foveal feedback for the action. The smooth timing of these fixations implies that where to look is learned and forms a part of a script or 'schema' representing the stages of a task. ${ }^{9}$ Third, there are less common, 'look ahead' fixations that are not associated with the current action but may encode object positions into memory for use later. In some ways, these fixations are most similar to those recorded in traditional lab experiments, in that they reflect visual information processing in the absence of action. Pelz and Canosa ${ }^{10}$ have noted that look-ahead fixations during the everyday task of hand-washing are highly task specific, and have speculated that they act to 'stitch together' subtasks and provide continuity for the actor.

Land and Hayhoe $^{6}$ summarised the diverse fixation routines observed in natural tasks as belonging to four functional groups: 'locating', look-ahead fixations, which retrieve visual information; 'directing' fixations, which acquire position information for an action; 'guiding' fixations, which alternate between two objects being manipulated; and 'checking' fixations which monitor whether a condition in the scene has been met (such as whether the kettle is full). In each of these cases the duration and frequency of fixations are distinct. The precise location of the fixation on an object also depends on the task and the action being performed. For example, although people tend to saccade straight to the centre of a picture of an object, where they can recognise it most efficiently, ${ }^{11}$ when planning a reach other object features may be more important (see Figure 1).

\section{Walking}

When walking around the natural environment we must coordinate our movements over variable terrain in the presence of many other objects. Tracking the eyes in this situation can provide some insight into how we pay attention in a complex environment. Of course, it is much easier to study this process by showing stationary participants images or video in a controlled laboratory setting, but such an approach has been increasingly criticised because it misses out on the realistic, actionbased context in which eye movements operate. ${ }^{12}$ In a study performed at the University of British Columbia, my colleagues and I investigated the differences between eye movements made while walking and those made while watching a video of the same route. ${ }^{13}$ Some items were fixated frequently in both situations, but there were also differences. For example, people walking in the real world spent more time looking at the path in front of them than did people watching the video (see Figure 2a).

Looking at the path in the real world is presumably important for planning locomotion. The timing of fixations relative to footsteps fits nicely with findings from the study of everyday tasks. When walking over irregular spaces, people look approximately two steps ahead, fixating the ground that will be traversed up to $1 \mathrm{~s}$ later. ${ }^{14,15}$ The function of these fixations must be to monitor the changing surface, acquiring this information in time for the motor system to adjust the body and limb movements. Gaze is then maintained by saccades and pursuit eye movements, as well as by the movement of the head.

Indeed, in our study large changes in gaze were normally accomplished by head movements, with eye
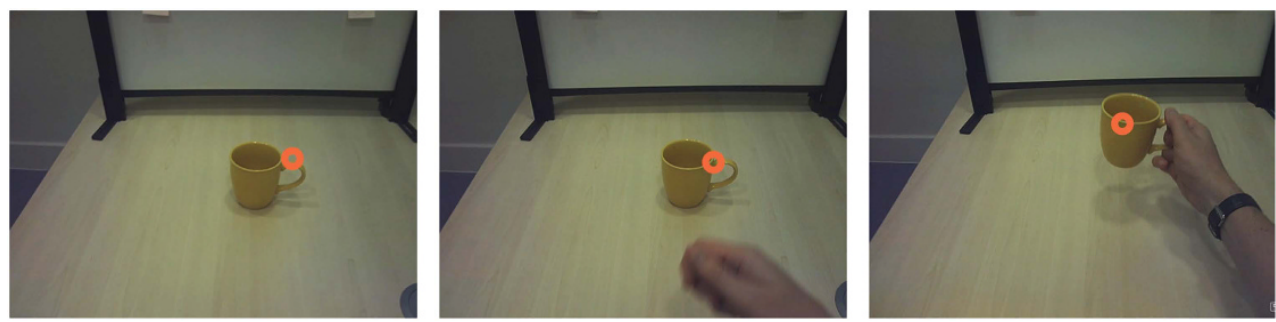

Figure 1 The view in front of an observer, with his point of gaze (circle) while picking up a mug. He fixates immediately on the handle, about $0.5 \mathrm{~s}$ before reaching for the object. Once lifted, gaze moves to a different location. 


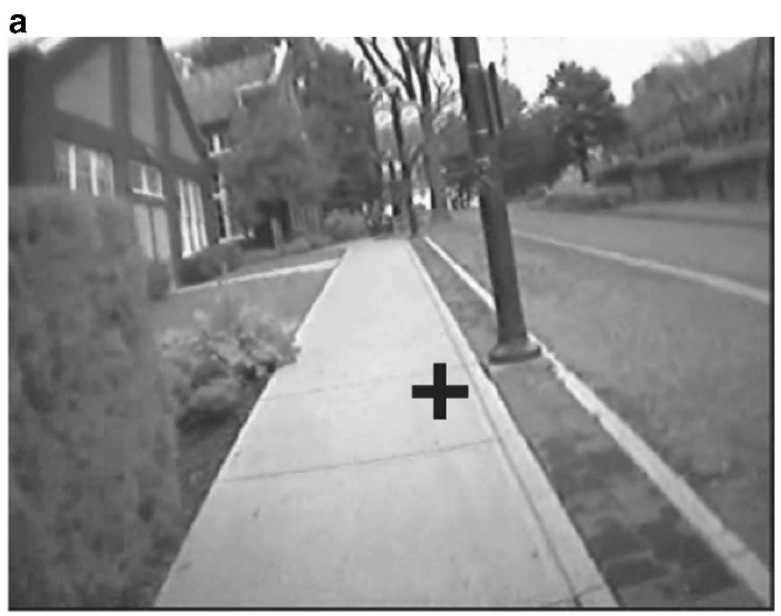

b

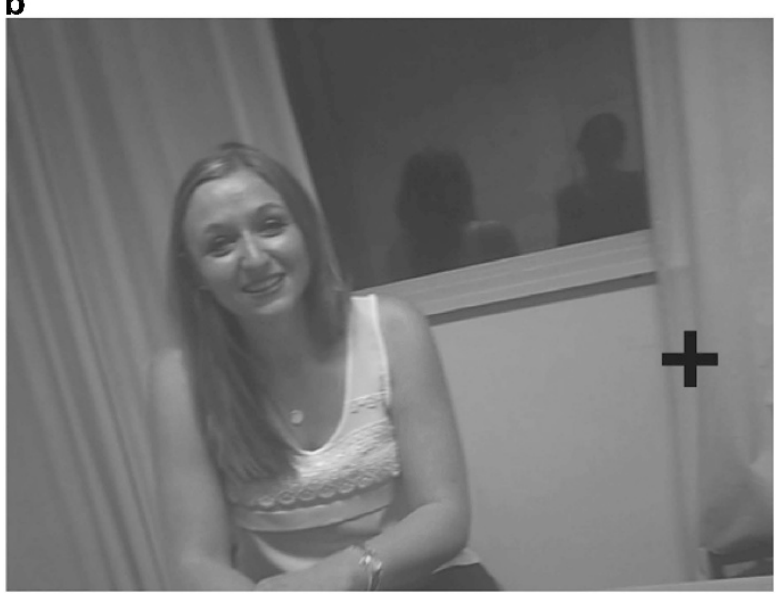

Figure 2 Two examples where directing gaze to visually inconspicuous items accomplishes different functions. When walking, fixating the path helps guide locomotion (a). When conversing, averting gaze is used as a signal while thinking and speaking (b).

position remaining within the central $10^{\circ}$ for most of the time. ${ }^{13}$ The coordination between the head and the eye, particularly for large gaze shifts, is the norm in everyday behaviour and hints at the neural links between eye movement control and the motor system more generally. If the function of fixations on the path during walking is to acquire information for planning footsteps, then we might expect changes in their distribution when the environment is more uncertain. Sure enough, when different road surfaces are compared, participants spend more time looking down on uneven steps than on a smoother road. ${ }^{16}$ Again, this change in sampling behaviour is brought about via changes in both head and eye position, with the head pointing lower (relative to the world), and the eyes looking downward (relative to the head).

Although measuring eye movement velocity in the real world remains difficult, 't Hart and Einhäuser's results show a pattern whereby people make slow eye movements downward, to track the approaching path, followed by faster, larger saccades upward to a new point. ${ }^{16}$

\section{Interacting with others}

When walking around the world we frequently look at other people, particularly when they are far away. ${ }^{13}$ However, moving our eyes in response to another person often has a separate function, distinct from taking in visual information, because eye gaze is a communicative signal. ${ }^{17}$ This explains why we readily turn towards and fixate a picture of a person, but avoid looking at the same person if they are physically present and able to see us. ${ }^{18}$ In the latter case, people are sensitive to the fact that looking at a stranger may be judged as confrontational or inappropriate. Let us consider two facets of how eye movements help us to interact with each other in everyday life.

First, eye gaze provides a signal of where somebody is attending, and this signal is readily picked up by other observers. In laboratory experiments, participants are quicker to react to stimuli that appear at a location being looked at by a schematic face. ${ }^{19}$ This 'gaze following' effect is largely automatic, develops early and is associated with sophisticated neural mechanisms. ${ }^{20,21}$ However, more realistic investigations of gaze following in everyday tasks have demonstrated that whether or not we track and follow the gaze of another person depends on the socio-communicative context. For example, we track and follow the gaze of others more readily when we are behind them (presumably because we ourselves cannot be seen). ${ }^{22}$ Gaze cues in a realistic building task are followed only when the accompanying spoken language is ambiguous. ${ }^{23}$

Second, even when we are not explicitly interested in the target of somebody's gaze, their eye position may function as a social signal. During conversation, we take turns in speaking - a sophisticated 'dance' in which gaze cues have an important role. ${ }^{17}$ We tend to look more at someone when we are listening than when we ourselves are talking. ${ }^{24}$ The function of fixations away from the face is quite different from those involved with picking up visual information (see Figure 2b). Deliberately averting gaze may facilitate thinking, ${ }^{25}$ but it also sends a signal to the conversant that it is not yet their time to speak.

Making regular eye movements to and away from the listener ensures that they are paying attention, as well as signalling that the speaker knows this (communication which may be more difficult via video or webcam ${ }^{24}$ ).

These examples illustrate that eye movements in social interaction function to both enhance visual perception of social cues (for example, when fixating someone's face) and to broadcast a social signal (for example, when making and breaking eye contact during a conversation). 
It is interesting to note that patients with strabismus may have particular difficulty in social settings, precisely because they are unable to initiate normal gaze cues. A study with healthy participants has confirmed that at least some gaze following takes place in response to misaligned eyes. ${ }^{26}$ However, Babar et $a l^{27}$ have shown that patients with strabismus perceive eye contact differently, particularly when this has resulted in one strongly dominant eye. Understanding the giving and receiving of gaze signals in this condition is therefore important when considering the psychosocial implications of corrective surgery.

\section{Conclusions}

I have described some patterns of fixations and saccades that are observed during complex, natural tasksinteracting with objects, walking around and speaking with other people. The eye movements in each case are different, as are their functions. When completing sequential tasks or moving around in the real world, we fixate in order to plan the current action. These fixations are highly specific, targeting the features necessary for moving our bodies and minimising the need to represent the environment in memory. During locomotion, the interplay between head and eye movements is complex and involves saccades, smooth pursuit eye movements and the vestibulo-ocular reflex. When interacting with others, eye movements serve an additional purpose as a communicative signal. This wide variety of functions suggests that learned eye movement routines are inseparable from the everyday tasks that make up natural human behaviour.

\section{Conflict of interest}

The author declares no conflict of interest.

\section{Acknowledgements}

I am grateful to Ben Tatler for introducing me to the symposium, and the organisers for their interest and encouragement.

\section{References}

1 Land MF. Motion and vision: why animals move their eyes. J Comp Physiol A 1999; 185: 341-352.

2 Gilchrist ID, Brown V, Findlay JM. Saccades without eye movements. Nature 1997; 390: 130-131.

3 Liversedge SP, Findlay JM. Saccadic eye movements and cognition. Trends Cogn Sci 2000; 4: 6-14.

4 Hayhoe M, Ballard D. Eye movements in natural behavior. Trends Cogn Sci 2005; 9: 188-194.
5 Land M, Tatler B. Looking and Acting: Vision and Eye Movements in Natural Behaviour. Oxford University Press: Oxford, UK, 2009.

6 Land M, Hayhoe M. In what ways do eye movements contribute to everyday activities? Vision Res 2001; 41: 3559-3565

7 Land M, Mennie N, Rusted J. The roles of vision and eye movements in the control of activities of daily living. Perception 1999; 28: 1311-1328.

8 Hayhoe M, Shrivastava A, Mruczek R, Pelz JB. Visual memory and motor planning in a natural task. J Vis 2003; 3: 6.

9 Land M. Vision, eye movements, and natural behavior. Vis Neurosci 2009; 26: 51-62.

10 Pelz JB, Canosa R. Oculomotor behavior and perceptual strategies in complex tasks. Vision Res 2001; 41: 3587-3596.

11 Foulsham T, Kingstone A. Optimal and preferred eye landing positions in objects and scenes. Q J Exp Psychol 2013; 66: 1707-1728.

12 Tatler BW, Hayhoe M, Land M, Ballard DH. Eye guidance in natural vision: reinterpreting salience. J Vis 2011; 11: 5.

13 Foulsham T, Walker E, Kingstone A. The where, what and when of gaze allocation in the lab and the natural environment. Vision Res 2011; 51: 1920-1931.

14 Patla AE, Vickers JN. How far ahead do we look when required to step on specific locations in the travel path during locomotion? Exp Brain Res 2003; 148: 133-138

15 Hollands MA, Marple-Horvat DE. Coordination of eye and leg movements during visually guided stepping. J Motor Behav 2001; 33: 205-216.

16 't Hart B, Einhäuser W. Mind the step: complementary effects of an implicit task on eye and head movements in real-life gaze allocation. Exp Brain Res 2012; 223: 233-249.

17 Kleinke CL. Gaze and eye contact: a research review. Psychol Bull 1986; 100: 78.

18 Laidlaw KE, Foulsham T, Kuhn G, Kingstone A. Potential social interactions are important to social attention. Proc Natl Acad Sci USA 2011; 108: 5548-5553.

19 Friesen CK, Kingstone A. The eyes have it! Reflexive orienting is triggered by nonpredictive gaze. Psychon Bull Rev 1998; 5: 490-495.

20 Emery NJ. The eyes have it: the neuroethology, function and evolution of social gaze. Neurosci Biobehav Rev 2000; 24: 581-604.

21 Pelphrey KA, Morris JP, McCarthy G. Neural basis of eye gaze processing deficits in autism. Brain 2005; 128 1038-1048.

22 Gallup AC, Chong A, Couzin ID. The directional flow of visual information transfer between pedestrians. Biol Lett 2012; 8: 520-522

23 Macdonald RG, Tatler BW. Do as eye say: gaze cueing and language in a real-world social interaction. J Vis 2013; 13: 6.

24 Freeth M, Foulsham T, Kingstone A. What affects social attention? Social presence, eye contact and autistic traits. PLoS One 2013; 8: e53286.

25 Doherty-Sneddon G, Bruce V, Bonner L, Longbotham S, Doyle C. Development of gaze aversion as disengagement from visual information. Dev Psychol 2002; 38: 438.

26 Hietanen J, Yrttimaa K. Where a person with a squint is actually looking: gaze-cued orienting in crossed eyes. Visual Cogn 2005; 12: 117-126.

27 Babar S, Khare GD, Vaswani RS, Irsch K, Mattheu JS, Walsh $\mathrm{L}$ et al. Eye dominance and the mechanisms of eye contact. J AAPOS 2010; 14: 52-57. 